\title{
Kardiodiagnostiktage 2021: Digital gerüstet für die Zukunft
}

Vom 4. bis 6. Februar 2021 fanden die 13. Deutschen Kardiodiagnostik-Tage 2021 kombiniert mit dem 14. Leipziger Symposium Nichtinvasive Kardiovaskuläre Bildgebung in Form eines besonderen Hybrid-Formats als Antwort auf die Corona-Pandemie statt. Im Interview zieht der wissenschaftliche Leiter der Veranstaltung, Prof. Dr. Matthias Gutberlet, eine Fortbildungsbilanz in Zeiten von COVID-19.

Herr Professor Gutberlet, auch die Kardiodiagnostik-Tage mussten sich der Pandemie beugen und auf ein digitales Konzept umstellen. Weshalb war es Ihnen wichtig, die Kardiodiagnostik-Tage in jedem Fall stattfinden zu lassen?

Die Veranstaltung hat sich nach einer anfänglich alle zwei Jahre stattfindenden Veranstaltung als jährliche Veranstaltung etabliert und fand nun schon zum 10. Mal unter inter-

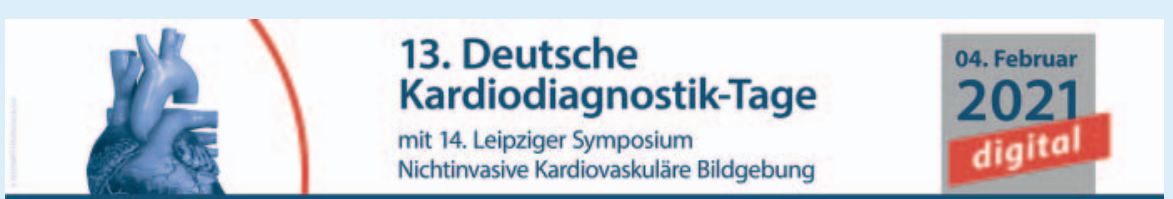

disziplinärer Leipziger Leitung statt. Die Veranstaltung ist zu einem wichtigen Treffpunkt für den wissenschaftlich-praktischen Austausch geworden, vor allem für die deutschsprachige radiologisch-nuklearmedizinische, aber auch die kardiologische „Community“, getragen von der AG Herz- und Gefäßdiagnostik in der DRG, sowie wichtigen Protagonisten der DGK und DGN auf dem Gebiet der nicht-invasiven kardiovaskulären Diagnostik. Die thorakale Bildgebung und potenzielle kardiovaskuläre Begleitschäden spielen bei PatientInnen während oder nach einer durchgemachten COVID-19-Infektion eine große Rolle. Des Weiteren stellen gerade die in den letzten zwei Jahren durch die neuen ESC-Leitlinien 2019 zum chronischen Koro- narsyndrom (CCS) und 2020 zum akuten Koronarsyndrom (ACS) aufgewerteten nichtinvasiven bildgebenden Verfahren zur Diagnose und Differentialdiagnose der koronaren Herzkrankheit auch unter Pandemiebedingungen einen Vorteil gegenüber den invasiven Maßnahmen dar. Deshalb mussten wir diese Themen unbedingt für unsere „Peer Group“ behandeln und die DKDT 2021 stattfinden lassen.

Wie haben Sie das Programm gestaltet und wie fällt ihre Bilanz aus?

Es war ein auf einen Tag reduziertes wissenschaftliches Programm aus drei Themenblöcken: 
1. Die neuen 2020er ESC-Leitlinien zum akuten Koronarsyndrom beim NSTEMI.

2. COVID-19 und das Herz.

3. Meine wichtigsten Studien aus 2020 aus Modalitätensicht.

Kombiniert haben wir diese Themenblöcke mit dem etablierten Format der FACE-OFF Session MRT, bei der zwei Kardio-MRT-Fälle mittels verschiedener Postprocessingsoftwares live ausgewertet wurden. Diesmal wurden ein Patient mit kardialer Beteiligung im Rahmen eines Long-COVIDSyndroms und ein Patient mit einem angeborenen Symptomkomplex aus bikuspider Aortenklappe, Isthmusstenose und Ebsteinanomalie vorgestellt. Zusätzlich wurde das Programm durch 4 verschiedene Industriesymposien aufgelockert - unter anderem fand der traditionelle modalitätenübergreifende Perfusionsworkshop statt. Dieses verkürzte Format hat online erstaunlich gut funktioniert, auch die FACE-OFF Session, was ich erst nicht so erwartet hatte.

\section{Auch die Q-Kurse zur Herzbildgebung fan- den digital statt. Wie wurde das Angebot angenommen?}

Das war auch einer der Hauptgründe, warum wir die DKDT 2021 unbedingt durchführen wollten: Viele junge, sich in Weiterbildung befindende KollegInnen, hatten sich über die Jahre diesen Termin vorgemerkt, um sich auf dem für RadiologInnen immer wichtiger werdenden Gebiet der kardiovaskulären Bildgebung fort- und weiterzubilden. Die Nachfrage hat uns positiv überrascht, da ja bereits im November 2020 im Rahmen des RÖKO DIGITAL 4 Q-Kurse stattgefunden hatten. Der Bedarf war und ist also weiterhin groß. Da parallele Veranstaltungen online nicht so gut funktionieren, wurden die Q1- und Q2-Kurse Kardio-CT und Kardio-MRT jeweils auf zwei Wochenenden im Anschluss an die DKDT 2021 Auftaktveranstaltung verteilt.

\section{Welche Erkenntnisse nehmen Sie für 2022 mit?}

Die Veranstaltung ist insgesamt sehr gut angenommen worden und hat mit deutlich über 600 TeilnehmerInnen allein für das wissenschaftliche Programm - plus jeweils fast 300 und teilweise sogar über 300 Teilnehmenden in den 4 Q-Kursen - die letzte Präsenzveranstaltung 2020 noch getoppt. Es gab auch einige technische Unzulänglichkeiten bei zwei parallel stattfindenden Industriesymposien, da haben wir dazugelernt. Insgesamt kam die Veranstaltung sehr gut an, vor allem, wie gesagt, die zertifizierten Q-Kurse. Das Onlineangebot werden wir also sicher auch bei der hoffentlich 2022 wieder möglichen Präsenzveranstaltung als zusätzliche Möglichkeit für Teilnehmerlnnen beibehalten müssen, die nicht nach Leipzig anreisen können oder wollen. Die Q1- und Q2-Kurse wurden von vielen Teilnehmenden zusammen gebucht, sodass wir in Zukunft den Q2-Kurs verkürzen können, wenn gemeinsame Inhalte nur einmal im Block behandelt werden. Wir freuen uns somit auf ein Wiedersehen in Leipzig 2022! 